\title{
Tendencias e instrumentos para identificar la sostenibillidad en la agroindustria
}

\section{Trends and tools for identifying strong and weak sustainability in agribusiness}

\author{
Gómez-Rodríguez, D. T; Editor Académico Dr. Roberto A. Berrios- \\ Zepeda
}

\author{
D. T Gómez-Rodríguez \\ dustin.tgr@gmail.com \\ Universitaria Agustiniana, Colombia \\ Editor Académico Dr. Roberto A. Berrios-Zepeda \\ roberto.berrios@ce.unanleon.edu.ni \\ Universidad Nacional Autónoma de Nicaragua, León, \\ Nicaragua
}

Revista Iberoamericana de Bioeconomía y Cambio Climático

Universidad Nacional Autónoma de Nicaragua, León, Nicaragua ISSN-e: 2410-7980

Periodicidad: Semestral

vol. 7, núm. 14, 2021

czuniga@ct.unanleon.edu.ni

Recepción: 10 Octubre 2021

Aprobación: 25 Noviembre 2021

URL: http://portal.amelica.org/ameli

jatsRepo/394/3941761010/index.html

DOI: https://doi.org/10.5377/ribcc.v7i14.12819

\begin{abstract}
Resumen: El objetivo general del artículo fue caracterizar las tendencias globales de la agroindustria, así como identificar algunos instrumentos que se pueden utilizar para medir la sostenibilidad fuerte como débil en aras del desarrollo sostenible eje trasversal de Agenda 2030 y los Objetivos de Desarrollo del Milenio-ODS. La metodología fue de corte cualitativo y el método fue de revisión documental. La conclusión principal, es que la agroindustria tiene mayor relevancia en el desarrollo de las sociedades, dado su relación directa e indirecta con la obtención de calorías por parte de los animales humanos y no humanos. Acotando que las tres tendencias identificadas en las que se desenvuelve son la Bioeconomía, la Biotecnología y la Agroindustria 4.0.
\end{abstract}

Palabras clave: Agroindustria, Bioeconomia, Biotecnología, Desarrollo Sostenible, Economía Rural, Economía Ecología.

\begin{abstract}
The general objective of the article was to characterise global trends in agribusiness, as well as to identify some instruments that can be used to measure both strong and weak sustainability in the interests of sustainable development crosscutting axis of agenda 2030 and the Millennium Development Goals (MDGs). The methodology was qualitative and the method was documentary review. The main conclusion is that agribusiness has greater relevance in the development of societies, given its direct and indirect relationship with the obtaining of calories by human and non-human animals. The three identified trends in which it develops are the Bioeconomy, Biotechnology and Agribusiness 4.0.
\end{abstract}

Keywords: Agro-industry, Bioeconomy, Biotechnology, Sustainable development, Rural Economy, Ecological Economy.

\section{INTRODUCCIÓN}

El intercambio incesante de diferentes capitales (social, financiero, intelectual, relacional, estructural) en un mundo que tiende a ser globalizado establece que los agentes económicos como los actores sociales reflejen estrategias competitivas para poder adaptarse a los tiempos del presente (Laverde, Almanza, Gómez, \& Serrano, 2020; Rubio, Téllez \& Gómez, 2019). Un ejemplo de ello es la agroindustria. Que es el conjunto de producción y transformación agrícola que comprende no solo las actividades propias agropecuarias, sino que 
se sintoniza con las acciones manufactureras que se suscriben en las maquinas, los fertilizantes, los pesticidas, las semillas (ANDI,2018). En suma, la agroindustria es la transformación de productos derivados de la pesca, la agricultura y las actividades forestales (OCDE \& FAO ,2019;FAO, 2018; Machado, 2002).

La agroindustria se ha diversificado a medida que pasa el tiempo, en virtud que un principio se basaba en el proteccionismo que le ofrecían algunos países y ahora se suscribe desde la liberación económica .En efecto, la industria alimentaria, por ejemplo, se ha transformado a lo largo de sus cadenas de suministros, así como, sobresalen gigantes trasnacionales que administran grandes extensiones de tierra. En particular, la incursión de monocultivos por medio de la agricultura industrializada como automatizada ha promovido la digitalización de la gestión agrícola y la agricultura de precisión siempre y cuando se tenga el respaldo abundante de capital como de grandes superficies (Fundación Heinrich Böll \& Fundación Rosa Luxemburgo ,2019; Harwood, J. ,2018; Kastner, Erb \& Haberl, 2014; FAO ,2009).

Por otra parte, dado el agotamiento como la relevancia del "oro azul" (agua), la agroindustria en su conjunto se ha relacionado fuertemente con diferentes instituciones del orden nacional como internacional para que el "oro azul" tenga un precio y así que el derecho de la población por el mismo se establezca desde el mercado. Sin olvidar, que desde el lado agropecuario (aunque la bioeconomía y las biotecnologías han emergido como tendencias de la agroindustria a nivel global) se evidencia por ejemplo, que los animales no humanos modificados genéticamente tienden a ser más enfermizos y en muchos casos son difíciles de comercializar , pero se ha acrecentado el número de laboratorios dedicados a estos nuevos procesos para seguir industrializando la ganadería (Rendón, 2017; Kastner, T., Rivas, M., Koch, W., \& Nonhebel, S. 2012; Heras, 2010;Larrión, 2011).

De ahí que en el mundo "Cuatro transnacionales dominan la importación y exportación de estas materias primas agrícolas: Archer Daniels Midland, Bunge, Cargill y la Louis Dreyfus Company. Juntas son conocidas como el Grupo ABCD o, simplemente, como ABCD. Archer Daniels Midland (abreviado, a su vez, como ADM), Bunge y Cargill son empresas estadounidenses. Louis Dreyfus tiene su sede en la capital de los Países Bajos, Ámsterdam. Todas fueron fundadas entre 1818 y 1902, y con excepción de ADM, siguen bajo la influencia de las familias fundadoras. Comercian y transportan, y también procesan muchas materias primas. Las transnacionales son dueñas de buques marítimos, puertos, ferrocarriles, refinerías, silos, molinos de aceite y fábricas. Su participación en el mercado mundial es de alrededor de $70 \%$ Fundación Heinrich Böll y Fundación Rosa Luxemburgo ,2019 p.28).

El objetivo general del artículo es caracterizar las tendencias globales de la agroindustria, así como identificar algunos instrumentos que se pueden utilizar para medir la sostenibilidad fuerte como débil en aras del desarrollo sostenible eje trasversal de Agenda 2030 y los Objetivos de Desarrollo del Milenio-ODS. Para ello, inicia con introducción ya desarrollada, prosigue con las tendencias globales que refleja la agroindustria. Continua con las líneas argumentativas de la categoría sostenibilidad y los instrumentos identificados para medir sí es Débil o Fuerte y termina con unas breves conclusiones.

\section{Materiales y MÉTODOS}

La metodología del artículo es de corte cualitativo y se tomó el método de revisión documental por medio de matrices en Excel, como el período estudiado es el siglo XXI (Gómez, Aldana \& Rodríguez, 2021; Gómez , Carranza \& Ramos 2017). La revisión documental tuvo como objetivo general identificar las simetrías y las asimetrías de los autores identificados a través de una una matriz en Excel en la que hay unas columnas que inician con la identificación del objetivo general del artículo consultado ,prosigue con la identificación de la metodología, las palabras claves, los resultados del ejercicio investigativo, las conclusiones del investigador y por último la síntesis del documento por pate del autor del presente escrito y con el cual se sostiene el mismo. De igual manera, el presente artículo tiene un enfoque descriptivo exploratorio (Guirao-Goris, 2015; Uribe,2011;Paramo,2008) y tuvo una ventana de observación del siglo XXI como se consultó en bases de 
datos avaladas ${ }^{[1]}$ a nivel a internacional como nacional (Aguilera ,Rincón \& Gómez, 2020). Entre las que cabe mencionar están Scopus, Wos ,Scielo, Redalyc, Pro Quest entre otras.

\section{Resultados}

\section{Tendencias de la agroindustria}

Las diferentes y cambiantes formas del consumo a nivel mundial han contribuido a que los agentes económicos busquen estrategias para posicionarse, adaptarse y replantear sus maneras de negocios. Precisamente, la agroindustria a nivel mundial en su afán de mantenerse en el mercado refleja tendencias que son maneras de competir en un mundo que tiende a ser globalizado (OCDE \& FAO 2019; Jacobi et al., 2018). Por consiguiente, se describen a continuación cada una de ellas iniciando en un primer lugar con la Bioeconomía y terminando con la agroindustria 4.0.

\section{Bioeconomía}

La Bioeconomía desde los derroteros de la narrativa neoliberal es una corriente de la economía que emergió desde la década de 1990 en EE. UU y en la Unión Europea- UE después del siglo XX que representa la mirada de una sociedad futura que sea menos dependiente de los recursos fósiles (Gómez \& Rincón ,2018). En este contexto (de la economía), la Bioeconomía esta cimentada en los usos de la biomasa y la oportunidades que nacen del diálogo entre la nueva biología y otras ciencias asociadas (Gómez, 2021c; Trigo, Henry, Sanders, Schurr, Ingelbrecht, \& Rocha, 2014; Trigo \& Henry, 2011).

La Bioeconomía desde la Nueva Economía es un diálogo entre las ciencias biológicas, las agrícolas, la agronomía, la biotecnología, la biología, la producción y la transformación sostenible de la biomasa renovable. Es decir, la comunión entre la biomasa y el conocimiento avanzado para su mejor explotación. Desarrollo fomentado en laboratorios que va en concordancia con las tendencias de la agroindustria porque pueden generar Bionegocios con posibles productos más competitivos y desde un sistema institucional sincronizado (Gomez et al., 2016;Trigo \& Harry, 2011;Gómez, 2021b; OCDE \& FAO ,2019; Vilella,2018; Zuniga et al., 2014; Trigo, 2011))

De ahí que tanto el "Programa de Bioeconomía y Desarrollo Productivo" del Instituto Interamericano de Cooperación para la agricultura (IICA) como la FAO consideren la Bioeconomía como una tendencia de económica social y política de la agroindustria, dado que entre 20 a 30 años se estima que la población mundial aumentará en 9.000 millones de personas que necesitan alimentarse, así como crecerá la demanda de alimentos en por lo menos del 50\% al 70\%.Sin olvidar, que existe evidencia de agotamiento de los recursos naturales, de reducción de las reservas del petróleo y sus derivados como se ha evidenciado impactos por el cambio climático ( FAO,2017; 2013; Trigo, 2012; IICA, 2011).

\section{Agroindustria 4.0}

Los cambios que ha generado la cuarta revolución industrial han contribuido en propiciar una de las tendencias de la agroindustria como lo es la Agroindustria 4.0. La primera Revolución Industrial se puede identificar entre los años 1760-1840 y su medio fue la invención del moto a vapor. La Segunda Revolución Industrial 2.0 tiene como derrotero la utilización de la electricidad y las cadenas de montaje. La Tercera Revolución Industrial es gracias a lo automatización de los procesos por medio de a los ordenadores y la Cuarta Revolución Industrial que se basa en la Revolución Digital (Arce,2020; OCDE \& FAO ,2019; 
Schwab, 2016). La agroindustria 4.0, es la forma como la misma se adapta a los cambios del mercado ( En virtud que este más sensibilizado, activo, informado como hiperconectado). Por consiguiente, la agroindustria se nutre de la utilización del BIG Data y la Agricultura de Precisión como una forma de responder a un mundo cambiante (Domene, 2018; Palacín, 2018; Martínez ,2018).

\section{Biotecnología}

La búsqueda de soluciones a los problemas agrícolas como pecuarios ha proporcionado sinergias entre la academia y el cambio tecnológico (Manzanelli 2012). Las tendencias en la tecnología agropecuaria como su trasferencia están cambiando la gestión ,la administración como la producción agropecuaria (Vilaboa \& Rivera ,2009).Aunque esta es una tendencia a largo plazo, dado las inversiones iniciales en la gran mayoría de los capitales y sobre todo su absorción cultural, se puede afirmar que es una tendencia en la agroindustria, dado su dinamismo como los resultados que exhiben los países con alto impacto biotecnológico (Milán $\&$ Zúniga,2021; OCDE \& FAO ,2019;EU COMMISSION ,2002;2004;2007;2010; Trigo \& Villareal,2009).

En consecuencia, las tres tendencias identificadas en el presente escrito de la agroindustria del presente se son la Bioeconomía, la biotecnología y la agroindustria 4.0.El siguiente apartado, continua la contextualización de la sostenibilidad fuerte y débil y cómo esta se relaciona con la agroindustria en aras de cumplir la Agenda 2030 y los Objetivos de Desarrollo Sostenible-ODS.

\section{Sostenibilidad}

En la década de 1960 incursionó en el mundo occidental una mayor conciencia frente a la relación entre la distribución , producción y transformación de bienes y servicios con el medio ambiente( López-et al., 2016). Es así como se han generado un sinnúmero de conferencias, informes entre entes multilaterales como entre países reunidos por la ONU ${ }^{[2]}$.Entre todos estos diálogos, el "Informe de Brundtland" es el documento por excelencia de una clase de sostenibilidad y fue publicado en el año 1987 que concibe que el desarrollo sostenible busca "asegurar que satisfaga las necesidades del presente sin comprometer la capacidad de las futuras generaciones para satisfacer las propias" (ONU, 1987, p. 29).Sin olvidar, que en este documento no existe diferencias entre sustentable y sostenible, ya que el error semántico correspondió más a las primeras impresiones de la categoría como sucedió en el caso mexicano que fue traducido del inglés "sustentable development" como desarrollo sostenible y en otros países de lengua española como desarrollo sustentable (Correa,2017;Dourojeanni,2000; Riechmaan, 1995.)

De igual manera, desde el punto de vista económico, la sostenibilidad se ha dividido en dos grandes núcleos con sus disciplinas que los sustentan .Por una lado, la Bioeconomía desde la Nueva Economía y la Economía Ambiental, que sostienen que el capital manufacturado puede suplir los servicios ecosistémicos del capital natural que es Sostenibilidad Débil y por el otro lado como discurso disidente la Economía Ecológica y la Economía Evolutiva que establecen todo lo contario, es decir, que el capital manufacturado no puede sustituir los servicios del capital natural y se le denomina Sostenibilidad Fuerte (Aguilera, Rincón \& Gómez, 2020; Aguilera,2018; Pavone, 2012; Correa, 2017; 2004;2003). 


\title{
Instrumentos de medición de sostenibilidad fuerte y débil
}

\author{
Sostenibilidad Fuerte-SF
}

\section{Huella ecológica-HE}

La Huella Ecológica estima o mide la “ carga” que tiene una población sobre su entorno inmediato natural, en términos de lo que necesita mantener la actividad económica (Correa,2017).Sobre todo, refleja el capital natural que demanda una economía por medio de la medición del cálculo de consumo de recursos y necesidades versus la capacidad de carga para asimilar los desechos como residuos frutos estos del resultado de la población establecida en el territorio ( Correa,2006;2004) .Por lo tanto, las categorías del uso de la tierra son 6, que componen el indicador que refleja los usos de la superficie productiva del planeta que son mutualmente excluyente (Arias,2006).

"el área o territorio ecológicamente productivo (cultivos, pastos, bosques o ecosistema acuático) necesaria para producir los recursos utilizados y para asimilar los residuos producidos por una población definida con un nivel de vida específico indefinidamente, donde sea que se encuentre esta área" (Wackernagel \& Rees, 1996, p.87).

Entre las críticas a este instrumento está la de (Van Hooter \& Bulte ,2000) que afirma que el HE puede ser una sobremedida del límite de sostenibilidad, ya que puede ser más efectivo controlar las emisiones de carbón que limitar la concentración en el ambiente a través de la captura de carbono. De igual manera, establecen dudas sobre la posibilidad de convertir efectivamente los flujo de residuos en relación con la tierra productiva, en virtud que el conocimiento sobre los efectos en el futuro (Correa,2017). También existen críticas del supuesto de elasticidad de sustitución completa entre las diferentes formas de la tierra (Wackernagel, 2009; Wackernagel, Onisto, Bello, Callejas, Linares, Méndez \& Suarez, 1999; Correa, 2017; Tobasura, 2008; Secretaría de Medio Ambiente y Recursos Naturales, 2012).

\section{Brecha Sostenible-BS}

Los primeros en generar el indicador de Brecha Sostenible fueron (Ekins \& Simon,1999). La BS tiene como función en primer lugar identificar el capital natural que debe de ser protegido, conservado y en qué grado. En segundo momento, se construyen indicadores que determinen sí los niveles se cumplen o que tan grande es la Brecha Sostenible. El tercer paso, es la cuantificación monetaria de los costos que incurre una sociedad para conseguir los niveles óptimos de sostenibilidad, para reducir la o las brechas de sostenibilidad. Las críticas que se le hacen es que es instrumento reduccionista y mecanicista (Neumayer,1999; Huerting \& Reijenders,2004;López,2012; Correa,2017).

\section{Flujos de materiales y de energía}

Material \& Energy, Flow Accounting- MEFA es un instrumento de contabilidad que busca identificar las entradas y salidas de materiales por unidad económica y al mismo tiempo genera indicadores agregados para dar a conocer el uso de los recursos como son la Extracción Domestica ED, el Consumo Doméstico (DM) y el Balance Comercial Físico BCF (Gómez,2021c;González de Molina, 2016; Eisenmenger, Ramos \& Schandl,2007). 


\section{Sin ,olvidar que dos formas de materiales para insumos no se pueden sumar sin el conocimiento de su impacto ambiental (Correa,2017;Neumayer,1999).}

\section{Sostenibilidad débil-SD}

\section{La Regla de Hartwick}

La Sostenibilidad Débil tiene como argumento que la tecnología y el capital manufacturado puede suplir los servicios ecosistémicos del capital natural. Los cual contribuye para afirmar que el capital reproducible es sustituible de modo que la economía no colapsaría cuando el recursos se agote (Correa,2004; Hartwcik, 1977). La Regla de Hartwick teniendo como derrotero la economía de los recursos, cuantifica la inversión en capital que se necesita para sopesar la reducción de los recursos no renovables. Dicha inversión, se efectúa para que el nivel de vida no se detenga a medida que la sociedad se desarrolla hacia un futuro indefinido (Solow,1974).

\section{Un recurso no renovable obtiene una renta neta de $R$ por año. $T$ años es el período en que se agota el recurso. El modelo que determina el ingreso sostenible del recurso, RI esec 1:}

$$
\mathrm{RI}=\mathrm{rRC}=r \sum \mathrm{RC}(1+r)=r\left(\sum(R-\mathrm{RI})(1+r)\right.
$$

T es cada uno de los momentos de la identidad matemática que refleja la duración del recurso agotable y el termino RI es el ingreso anual sostenible. El corchete ,refleja el capital disponible cuando el recurso que no es renovable se agote. Aunque es un instrumento muy interesante, se le critica el supuesto que establece que el capital natural su valor será constante y sobre todo la utilización de precios sombras, ya que se considera irreal. "estimar los precios sombra es difícil y complejo, además está sujeto a una gran incertidumbre” (Correa,2017, p.100). Por consiguiente, la Regla de Hartwick, solo es significativa sí se mantiene el supuesto del capital natural puede ser sustituible por capital manufacturado tanto en la función de utilidad como en la producción, es decir una creencia optimista en torno a las consecuencias del crecimiento económico (Neumayer, 1999; Tisdell, 1997; Turner, 1993).

\section{MESMIS}

La Evaluación del Sistema de Manejo de Recursos Naturales Incorporando Indicadores de SustentabilidadMESMIS es un método de análisis que se divide en 6 pasos. El número uno se basa en determinar el objeto de estudio, el segundo es comprobar puntos críticos, el tercero es la elección de indicadores estratégicos. El número cuatro es la medición y el monitoreo. El quinto es la presentación e integración de resultados y el sexto, son las conclusiones y las recomendaciones (Saravia ,1983; Decamino \& Müller ,1993). Entre las críticas que posee este marco evaluativo es que se necesita un grupo multidisciplinario para realizar la escogencia de indicadores y la segunda que se deben tener datos sin perturbaciones lo cual es bastante raro en lo rural (Barrezueta,2015). 


\section{SAFE}

SAFE en sus siglas en inglés (Sustainability Assesment Of Farming and the Enviroment Farmerwork) es un instrumento que se aplica en actividades agrarias por medio de la Teoría adaptada de PCI (Principio, Criterio, Indicador). El método se utiliza para evaluar a los bosques teniendo como línea los objetivos en los que se dimensione lo ambiental, lo social como lo económico. Así como, los principios donde se delimitan las condiciones normales para alcanzar la sostenibilidad. Continua con los criterios de los resultados de la agroindustria y termina con los indicadores que pueden ser cualitativos o cuantitativos que pueden ser evaluados en relación con los criterios de donde resulte los indicadores (Sanchez,2009; Vancauwenbegh, Biala, Bielders, Brouckaert, Franchois, García \& Peeters, 2007; Sarandón, Zuluaga, Cieza, Gómez, Janjetic \& Negrete, 2006).Las críticas que se han revisado son más del orden de la construcción de los indicadores en que se relacionan lo social, lo económico como lo ambiental (Fernández ,2006) y la selección de los indicadores (Barrezueta,2015;Cantún, Becker, Bedano, Schiavo \& Parra, 2009).

\section{Análisis de Ciclo de Vida-ACV}

El ACV es un método que evalúa los posibles impactos ambientales de un bien o un servicio durante su existencia como los son la extracción, la producción la distribución, uso y fin .Desde el punto de vista jurídico se rige con ISO 14040 e ISO 14044.Las fases del ciclo generalmente son cuatro: Definición del Objetivo y el Alcance, Inventario de Ciclo de Vida, Evaluación del Impacto de Ciclo de Vida e interpretación del Ciclo de Vida. Una de las posibles críticas que tiene este método es que no realiza una evaluación de riesgo, porque no utiliza la exposición, variable para analizar el riesgo. De igual manera, aunque la ACV mide las emisiones, no identifica el impacto real ya que depende de cuándo, dónde y cómo se generen en el ambiente (Dahllöf, 2003; Monroy, Van Hoof \& espinosa,1999).

\section{Discusión}

La agroindustria a nivel mundial ha contribuido con la reconfiguración de los tejidos sociales, el aparato productivo y sobre todo con el medio ambiente tanto desde lo positivo como lo negativo. Las tendencias de la agroindustria se suscriben con las narrativas neoliberales de la Bioeconomía desde la Nueva Economía, las Biotecnologías y la Agroindustria 4.0 como sus mediciones en promedio se realizan desde la Sostenibilidad Débil. En efecto, los aportes del presente artículo señalan que la sostenibilidad depende tanto de las tasas de crecimiento como del capital natural y esto va en concordancia con el uso del capital humano y las tasas de reposición desde la sostenibilidad débil (Gómez, Barbosa \& Téllez, 2021;Dueñas, Ccasani, Huatuco, García \& Inga, 2019 ;González, Montes, Santos \& Monedero,2008; Gómez-Baggethum \& de Groot, 2007).

La Sostenibilidad Fuerte va en concordancia con los derroteros de la economía ecológica, ya que la economía ecológica argumenta que las actividades de los animales no humanos y humanos, no se encuentran en un vacío ontológico y biofísico, sino que hay intercambio en este caso del capital natural y la agroindustria (Maldonado ,2014;González de Molina, 2016;Maldonado,2021;Gómez, Barbosa \& Leuro, 2017; Gómez, Ariza \& Velasco, 2018). En particular, es imperativo utilizar otros conceptos ,otras categorías como métodos para poder contrarrestar los impactos negativos de la transformación productiva que efectúan los agentes económicos en el ambiente (Porter \& Heppelmann, 2016; Solís \& Burbano, 2015;Uribe et al,2011; Rendón \& Cardona, 2005). 


\section{Conclusiones}

El presente escrito es fruto de la tesis doctoral en desarrollo del autor titulada Metabolismo agrario de la agroindustria de la palma de aceite en el territorio de Aracataca Magdalena Colombia en el período 1965-2018 del doctorado en Agrociencias de la Universidad de la Salle dirigida por el PhD Jaime Alberto Rendón Acevedo.

La agroindustria tiene un gran impacto en las sociedades dado su relación directa con la obtención de calorías por parte de los animales humanos y los no humanos. Precisamente, los cambios incurridos en la producción, la transformación y la distribución desde lo pecuario, lo agrícola y lo acuicultor entre vendedores y consumidores finales han contribuido en la fusión entre organizaciones que son dueñas de las cadenas de valor. De ahí, que exista en el presente de la agroindustria una concentración de poder, una incesante reducción de costos, una mayor utilización de tecnologías, un gran apoyo por parte de diferentes gobiernos ,mayor dislocación de los procesos productivos que se han potencializado bajo tres tendencias claras como lo son: la Bioeconomía, la Biotecnología y la Agroindustria 4.0.

Las ciencias de la sostenibilidad han llegado para quedarse en la agroindustria dado sus tendencias y sus diferentes instrumentos para medirlas ya sea desde la Débil o la Fuerte. En efecto, la agroindustria genera impactos medioambientales y estos a su vez sociales.De ahí, la necesidad que la agroindustria identifique otros instrumentos, otras tendencias porque su actuar no se realiza en un vacío ontológico y bióticos sino todo lo contrario. Su desarrollo tiene un costo ambiental.

\section{ReFERENCIAS}

Aguilera M, Rincón H., \& Gómez, D.(2020). Bioeconomics, a research alternative in management and related fields. In M. Aguilera-Prado and H. Rincón Moreno (Eds.), Temas y métodos de investigación en negocios, administración, mercadeo y contaduría. Editorial Uniagustiniana. 193-225

Aguilera, M. (2018). Economía convencional y ecológica como simientes de propuestas contra el cambio climático. En Repensando el desarrollo: lecturas interdisciplinares (pp. 111-147). Bogotá, D. C.: Editorial Uniagustiniana.

Arce, S. (2020). La innovación agroindustrial: componentes, tendencias y acciones. Revista E-Agronegocios, 6(1).1-11 DOI: https://doi.org/10.18845/rea.v6i1.4938

Arias, F. (2006). desarrollo sostenible y sus indicadores. Documento de Trabajo. Núm. 93. CIDSE, Centro de investigaciones y documentación socioeconómica, Facultad de Ciencias sociales. Univalle.

Barrezueta, S. (2015). Introducción a la sostenibilidad agraria: Con enfoque de sistemas e indicadores. Ediciones UTMACH. Quito: Ecuador.

Cantún, M.., Becker, A.., Bedano, J.., Schiavo, H.., \& Parra, B. (2009). Evaluación del impacto del cambio de uso y manejo de la tierra mediante indicadores de calidad de suelo, Córdoba, Argentina. Cadernos Do Laboratorio Xeoloxico de Laxe, 34, 203-214.

Correa, F. (2017). Desarrollo sostenible. Revisión teórica desde la economía Medellín: Colombia. Ediciones UNAULA.

Correa, F. (2004). Economía de la sostenibilidad: perspectivas económicas y ecológicas. Ensayos de economía. 24 (14) 60-91.

Correa, F. (2003). Economía del desarrollo sostenible: propuestas y limitaciones de la teoría neoclásica. Semestre económico (12).

Dahllöf, L. (2003). Life Cycle Assesment (LCA) applied in the textiles sector: the uselfulness, limitation and methodological problems. A literatura Review.9: 16-32.

De Camino, R., \& Müller, S. (1993). Sostenibilidad De La Agricultura Y Los Recursos Naturales. (Instituto Interamericano de Cooperación para la Agricultura - IICA-, Ed.). San José, Costa Rica: Deutsche Geseltschaft fur Technisch Zusammenarbeit (GTZ) Gmbh 
Domene, A (2018) La aplicación del Big Data en el sector agroalimentario. Obtenido en: https:/futurizable.com/ big-data-agro/

Dueñas, A., Ccasani, J., Huatuco, M., García, V., \& Inga, D. (2019). Sostenibilidad de la Agroindustria Nacional en términos de recursos naturales. Campus, (28),157-182 https://doi.org/10.24265/campus.2019.v24n28.04

Eisenmenger, N., Ramos, J., \& Schandl, H. (2007). Análisis del metabolismo energético y de materiales de Brasil, Chile y Venezuela. Revista Iberoamericana de Economía Ecológica, 6: 17-39.

EU COMMISSION (2002). Life Science and Biotechnology-a Strategy for Europe. Communication from the Commission to the European Parliament, the Council, the Economic and Social Committee and the Committee of the Regions. Office for Official Publications of the European Communities. Obtenido en: http:// ec.europa.eu/biotechnology/pdf/com2002-27_en.pdf

EU COMMISSION (2004). Towards a European knowledge-based bioeconomy. DirectorateGeneral for Research and Innovation Office for Official Publications of the European Communities, Luxemburgo. Obtenido en: https://publications.europa.eu/ en/publication-detail/-/ publication/e0d4cc02-6da4-45b1-9535- 649664f97a6d

EU COMMISSION (2007). En route to the Knowledge based Bioeconomy. Bruselas. Obtenido en: https:// dechema.de/dechema_media/Downloads/Positionspapiere/Cologne_Paper.pdf

EU COMMISSION (2010). The Knowledge-based bioeconomy (KBBE) in Europe: achievements and challenges. Technical Reprot Clever Consult BVBA, European Research área. Obtenido en: http://www. mercadosbiotecnologicos.com/documents/the_knowledge_based_bioeconomy_kbbe_in_europe.pdf

Ekins, P., \& Simon, S. (1999). The sustainability gap: a practical indicator of sutainability in the framerwoek of the national accounts. Internatoional Journal of Sustainable Development. (2) 32-58 https://doi.org/10.1504/ IJSD.1999.004313

FAO. (2018). Organización de las Naciones Unidas para la Alimentación y la Agricultura. Organización de las naciones Unidad para la Alimentación y la Agricultura. Obtenido en: https//www.fao.org/docrep/w5800s/ w5800s $12 . h t m$

FAO, (2017). Agroindustria y concentración de la propiedad de la tierra. FAO. Obtenido en: https:// drive.google.com/file/d/0B6wJrsxILA_SmF2MC1ZTFA5T0E/view

FAO, (2013). Agroindustrias para el desarrollo. FAO. Obtenido en: http://www.fao.org/docrep/017/i3125s/ i3125s00.pdf

FAO. (2009). La agricultura mundial en la perspectiva del año 2050. Roma: CEPAL.

Fernández F. (2006). Aproximación a los indicadores de sostenibilidad y medio ambiente. In Indicadores de Sostenibilidad y Medio Ambiente: Métodos y Escala (pp. 23-71). Sevilla: Consejería del Medio Ambiente, Junta Andalucía.

Fundación Heinrich Böll \& Fundación Rosa Luxemburgo (2019). Atlas de la agroindustria. datos y hechos sobre la industria agrícola y de alimentaos. Ciudad de México, México: Greenprint.

Gómez, D., Barbosa, E. \& Téllez, C. (2021). Transitions against the Problems of the 21st Century the Ecological Economy. Asian Journal of Agricultural Extension, Economics y Sociology, 39(9), 76-84. https:// doi.org/10.9734/ajaees/2021/v39i930644

Gómez ,D., Aldana, K., \& Rodríguez, R. (2021). Antropologías del desarrollo, enfoques alternativos y postdesarrollo. Breve revisión de conceptos y apuntes críticos. Población y Desarrollo. 27 (52): 108 - 122. https:// doi.org/10.18004/pdfce/2076-054x/2021.027.52.108

Gómez, D. (2021a). Sostenibilidad. Apuntes sobre sostenibilidad fuerte y débil, capital manufacturado y natural. Inclusión y Desarrollo, 8 (1), pp 131-143 https://doi.org/10.26620/uniminuto.inclusion.8.1.2021.131-143

Gómez, D. (2021b). Biofuels. Lines of debate around the production ofagrofuels worldwide. Revista e-Agronegocios, 7(2),83-98 https://revistas.tec.ac.cr/index.php/eagronegocios/article/view/5688 https://doi.org/10.18845/ ea.v7i2.5688

Gómez, D. (2021c). Metabolismo social y bioeconomía Dialogo de saberes. Apuntes De economía Y Sociedad, 2(2),21-27. https://doi.org/10.5377/aes.v2i2.12807 
Gómez, D., Ariza, E., \& Velasco, N. (2018). Diálogos entre la economía ecológica y la bioeconomía. Bogotá: Colombia: Editorial Universidad de San Buenaventura. GKA Social 2019 VII Congreso Internacional de Ciencias Sociales. Université París Diderot, Francia. Julio 17-19. Ponencia.

Gómez, D., \& Rincón, H. (2018). La Bioeconomía como posible estrategia comparativa. Alianza del Pacífico: caso Colombia. Ciencias Económicas, 1, 101-115. https://doi.org/10.14409/rce.v1i0.7750

Gómez, D., Barbosa, E., \& Leuro, J. (2017). Ecología y Bioeconomía. El diálogo de saberes. Revista Clío en América.11 (21),108-119. https://doi.org/10.21676/23897848.2086

Gómez, D. T., Carranza, Y. \& Ramos, C. (2017). Revisión documental, una herramienta para el mejoramiento de las competencias de lectura y escritura en estudiantes universitarios. Chakiñan, Revista de Ciencias Sociales y Humanidades, (1), 46-56. https://doi.org/10.37135/chk.002.01.04

Gómez, D., Barbosa, E., \& Rojas, W. (2016). El Biodesarrollo como ruptura de la categoría desarrollo. Revista de ciencias económicas.2(13) 75-87. https://doi.org/10.14409/rce.v2i0.6433

González de Molina, M. (2016). Introducción al Metabolismo social y sus aplicaciones. Ponencia. Seminario sobre metabolismo Social. Universidad de Santiago de Chile, Santiago, 7 de noviembre de 2016. Obtendio en: https:// www.youtube.com/watch?v=2s3ETIqqrZM

Gómez-Baggethum, E., \& de Groot, R. (2007). Capital natural y funciones de los ecosistemas: explorando las bases ecológicas de la economía. ecosistemas. Revista científica y técnica de ecología y medio ambiente, 4-14.

González, J., Montes, C., Santos, I., \& Monedero, C. (2008). Invirtiendo en capital natural: un marco para integrar la sostenibilidad ambiental en las políticas de cooperación. ecosistemas. Revista científica de ecología y medio ambiente, 52-69.

Hartwick, J. (1977). Intergenerational Equity and Investing the Rents from Exhaustible Resources.American Economic Review, 66, 972-974.

Harwood, J. (2018). The green revolution is a process of global circulation: plants, people, and practices. Historia Agraria, 75. 7-31 https://doi.org/10.26882/histagrar.075e01h

Heras, M. (2010). Cuando el olmo pide peras. El insostenible consumo energético del sistema agroalimentario. Barcelona, España Edición: Asociación Catalana de Ingeniería sin Fronteras.

Huerting R., \& Reijndress, F. (2004). Broad sustainability contra sustainability: the proper construction of sustainability indicators: Eco-efficiency as an abandonment of the nature. Ecological Economics. 50(3-4) 249-260 https://doi.org/10.1016/j.ecolecon.2004.03.031

IICA. (2011). La innovación para el logro de una agricultura competitiva. IICA .Obtenido en: http://webiica.iica.ac.cr/organos_iica/COMITE/SysInfo_2011/documentos/-E\%20(DT-574)\%20Innovac-gricCompetit-sustentable.pdf

Jacobi, J., Mukhovi, S., Llanque, A., Augstburger, H., Käser, F., Pozo, C., ... \& Speranza, C. I.(2018). Operationalizing food system resilience: An indicator-based assessment in agroindustrial, smallholder farming, and agroecological contexts in Bolivia and Kenya. Land use policy, 79, 433-446. https://doi.org/10.1016/j.landusepol.2018.08.044

Kastner, T., Erb, K., y Haberl, H. (2014). Rapid growth in agricultural trade: effects on global area efficiency and the role of management. Environmental Research Letters 9(3): 034015.

Kastner, T., Rivas, M., Koch, W., \& Nonhebel, S. (2012). Global changes in diets and the consequences for land requirements for food. Proceedings of the National Academy of Sciences 109(18), 6868-6872. https:// doi.org/10.1073/pnas.1117054109

Laverde, M., Almanza, C., Gómez, D., \& Serrano, C. (2020). El Capital Relacional como Recurso Diferencial y Valioso para las Empresas. PODIUM, (37), 57-70. https://doi.org/10.31095/podium.2020.37.5

Larrión, J. (2011). Luces y sombras de la tercera revolución verde. Demandas sociales, opciones agrícolas y desacuerdos narrativos. Bitácota-e. Revista Electrónica Latinoamericana de Estudios Sociales, Históricos y Culturales de la Ciencia y la Tecnología. 13, 3-23

López, I. (2012). Sostenibilidad débil y fuerte y democracia deliberativa. el caso de la agenda local 21 local de Madrid. Tesis doctoral. Universidad Carlos III de Madrid. Obtenido en: https://e-archivo.uc3m.es/bitstream/ handle/10016/16270/tesis_lopez_pardo_2012.pdf 
López-González, A., Zuniga-González, C., Sol-Sánchez, Ángel, \& Santiváñez-Galarza, J. (2016). Teorías del desarrollo sustentable para el siglo XXI: un breve análisis. Revista Iberoamericana De Bioeconomía Y Cambio Climático E-ISSN 2410-7980, 2(1), 437-451. https://doi.org/10.5377/ribcc.v2i1.5710

Maldonado, C. (2021).Las Ciencias de la Complejidad son Ciencias de la Vida / Carlos Eduardo Maldonado - Primera edición. Chile: Trepen Ediciones.

Maldonado C. (2018). Bioeconomy, Biodevelopment and civilization. A map of problems and solutions. In Epistemologies of the South to germinate alternatives to development. Debate between Enrique Leff, Carlos Maldonado and Horacio Machado. Bogotá, Colombia: Editorial Universidad del Rosario. 57-81.

Maldonado C. (2014). Biodevelopment and complexity. Proposal of a theoretical model. In M. Eschenhaguen, A journey through alternatives to development: Perspectives and theoretical proposals. Bogotá, Colombia: Universidad del Rosario. 71-94.

Machado, A. (2002).de la estructura agraria al sistema agroindustrial. Bogotá, Colombia: Editorial Universidad Nacional de Colombia.

Martínez, D (2018) ¿Puede el Big Data mejorar la seguridad alimentaria de mi empresa? .Obtenido en: https:// www.ainia.es/tecnoalimentalia/tecnologia/bigfood-big-data-innovacion-empresas-agroalimentarias/

Milán Pérez, J., \& Zúniga- González, C. (2021). Necesidades de investigación y transferencia de tecnologías sobre cambio climático en Nicaragua: Una oportunidad en la Bioeconomía. Revista Iberoamericana De Bioeconomía Y Cambio Climático E-ISSN 2410-7980, 7(13), 1518-1543. https://doi.org/10.5377/ribcc.v7i13.11270

Monroy, N., Van Hoof, B \& Espinosa, J. (1999). LCA (Life Cycle Analysys). Una herramienta de la industria para conciliar la crisis ambiental y el desarrollo empresarial. Revista de ingeniería. 9:44-51

Neumayer, E. (1999). Weak versus strong sustainability. London, Ed Ward Elgars Publisher.

OCDE y FAO (2019). OCDE-FAO Perspectivas Agrícolas 2019-2028, OECD Publishing, París/Organización de las Naciones Unidas para la Alimentación y la Agricultura (FAO), Roma.https://doi.org/10.1787/7b2e8ba3-es.

ONU, (1987). Nuestro futuro común. Madrid: Alianza.

Pavone, V. (2012). Ciencia, neoliberalismo y bioeconomía. Revista Iberoamericana de Ciencia, Tecnología y Sociedad, $145-161$.

Paramo, P. (2008). La Investigación en las Ciencias Sociales. Técnicas de recolección de Información.: Bogotá, Colombia: Universidad Piloto de Colombia.

Porter, M. E., \& Heppelmann, J. E. (2016). How smart, connected products are transforming competition. Harvard business review, 92(11), 18-24.

Riechmann, J. (1995). De la economía a la ecología. Madrid: Trotta.

Ríos, L., Ortiz, M. \& Álvarez, J., (2005). Debates on sustainable development. Towards holistic view of reality, Environment, development, and sustainability. Environment, Development and Sustainability, 7(4): 501-518. https://doi.org/10.1007/s10668-004-5539-0

Palacín, T (2018) La industria alimentaria, estancada ante la digitalización. Obtenido en: de https:// www.innovaspain.com/la-industria-alimentaria-estancada-ante-la-digitalizacion/

Rendón, J. (2017). Pensar la economía en torno a la naturaleza. Prologo. En: Desarrollo sostenible. Revisión teórica dese a la economía. Ediciones UNAULA. Medellín: Colombia.

Rendón, J., \& Cardona, M. (2005). Reformas estructurales: modelos difíciles de armar. Revista Equidad y Desarrollo. (3), 9-33. https://doi.org/10.19052/ed.371

Rubio, G. A., Téllez, C. A., \& Gómez, D. T. (2019). Análisis de los factores que componen un sistema de gestión empresarial: estudio de caso para la dirección de vivienda. Revista Nacional De Administración, 10(2), 47-60. https://doi.org/10.22458/rna.v10i2.2730

Sánchez, G. (2009). Análisis De La Sostenibilidad Agraria Mediante Indicadores Sintéticos: Aplicación Empírica Para Sistemas Agrarios de Castilla y León. Universidad Politécnica de Madrid, Madrid.

Sarandón, S., Zuluaga, M., Cieza, R., Gómez, C., Janjetic, L., \& Negrete, E. (2006). Evaluación de la sustentabilidad de sistemas agrícolas de fincas en misiones, argentina, mediante el uso de indicadores. Agroecología (Vol. 1). La Plata 
Saravia, A. (1983). Un Enfoque de Sistemas para el Desarrollo Agrícola. San José, Costa Rica: IICA.

Secretaría de Medio Ambiente y Recursos Naturales. (2012). Huella ecológica, datos y rostros. Centro de Educación y Capacitación para el Desarrollo Sustentable. Obtenido en: https://www.sema.gob.mx/descargas/manuales/ HuellaEcologica_SEMARNAT.pdf

Schwab. (2016). La cuarta revolución industrial. Editorial Debate. Barcelona: España

Solow, R. (1974). Equidad intergeneracional y recursos agotables. Revisión de estudios económicos (simposio): 29-46.

Solís, S., \& Burbano, A. (2015) análisis de tendencias tecnológicas del sector agroindustrial en el departamento del cauca. (Trabajo de grado en ingeniería Agroindustrial) p.245 Universidad del Cauca. Popayán Cauca Colombia.

Tobasura, I. (2008). Huella ecológica y biocapacidad: indicadores biofísicos para la gestión ambiental. El caso de Manizales, Colombia. Revista Luna Azul.26, 119-136

Trigo, E., Henry, G., Sanders, J., Schurr, U., Ingelbrecht, I., \& Rocha, P. (2014). Hacia un desarrollo de la Bioeconomía en América latina y el caribe. En E. Hodson de Jaramillo, Hacia una Bioeconomía en América latina y el caribe en asociación con Europa (pp.17-43). Bogotá, Colombia: Editorial Pontificia Universidad Javeriana.

Trigo, E. (2011). Los nuevos escenarios para la institucionalidad de la investigación agroalimentaria en América latina. En E. Trigo, N. Mateo y C. Falconi, Innovación Agropecuaria en América Latina y el Caribe: Escenarios y Mecanismos Institucionales. Obtenido en: https://publications.iadb.org/ bitstream/handle/11319/5856/ Innovacion\%20Agropecuaria\%20en\%20 America\%20Latina\%20y\%20el\%20 Caribe\%20.pdf?sequence=1

Trigo, E. (2012). Los nuevos escenarios para la institucionalidad de la investigación agroalimentaria en América latina y el Caribe. Washington D.C., USA: Interamerican Development Bank.

Trigo, E., y Henry, G. (2011). A Bioeconomy for Latin America and the Caribbean: opportunities and challenges from a policy perspective. Bioeconomy Policy Note No 2011_01.

Trigo, E., \& Villareal. (2009). La agrobiotecnología en las Américas: una mirada a la situación actual y las tendencias futuras. Informe técnico. San José, Costa Rica: Instituto Interamericano de Cooperación para la Agricultura.

Tisdell, C. (1997). Capital natural resources substitution: The debate of Georgescu Roegen (Through Daly) with Solow/Stiglitz, Forum.Ecological Economics. (22), 289-291 https://doi.org/10.1016/S0921-8009(97)00089$\mathrm{X}$

Turner, R (1993). Sustainability: prnciples and tractice. En: Turner R., (edition). Sustainable Enviromne Economics and management: Prnciples and Practice. London: Belhaven Press, pp 3-36

Van Cauwenbergh, N., Biala, K., Bielders, C., Brouckaert, V., Franchois, L., García V., \& Peeters, A. (2007). SAFEA hierarchical framework for assessing the sustainability of agricultural systems. Agriculture, Ecosystems and Environment, 120(2-4), 229-242 https://doi.org/10.1016/j.agee.2006.09.006

Van Kooten, P., \& Butle, J. (2000). The economics of nature. Managing biological asset, London; Blackwell Publisher. 550.

Vilaboa, J., \& Rivera, P. (2009). Caracterización socioeconómica y tecnológica de los sistemas ganaderos en siete municipios del estado de Veracruz, México. Zootecnia Trop. 27(4): 427-436

Vilella, F. (2018) Bioeconomía: una oportunidad estratégica. Obtenido en: http://www.maizar.org.ar/vertext.php? $\mathrm{id}=602$

Uribe, J. (2011). La investigación documental y el estado del arte como estrategias de investigación en ciencias sociales. Páramo, O (Comp.). La investigación en ciencias sociales. Estrategias de investigación (195-210). Bogotá: Universidad Piloto de Colombia.

Uribe G. Fonseca R., Bernal, R., Contreras P., \& Castellanos, D. (2011) Sembrando innovación para la competitividad del Sector Agropecuario Colombiano. En: Ministerio de Agricultura y Desarrollo Rural (Colombia). Bogotá, Colombia Giro Editores Ltda. p.180.

Wackenagel, M., Onisto, L., Bello, P., Callejas, A., Linares, S., Médez, J. \& Suarez, M. (1999). National natural capital Accounting wuth the ecological footprint concept. Ecological Economics. (29)375-390 https:// doi.org/10.1016/S0921-8009(98)90063-5 
Wackernagel, M. (2009). La huella ecológica en un mundo con recursos limitados. Obtenido en: http://hdr.undp.org/ en/media/HD_Insights_September2009_SP.pdf.

Wackernagel, M. \& Rees, W. (1996). Our Ecological Footprint, Reducing Human Impact on the Earth. New Society Publishers, Philadelphia.

Zúniga-González, C. A., Durán Zarabozo, O., Dios Palomares, R., Sol Sánchez, A., Guzman Moreno, M. A., Quiros, O., \& Montoya Gaviria, G. D. J. (2014). Estado del arte de la bioeconomía y el cambio climático (No. 1133-2016-92457, pp. 20-329). DOI 10.22004/ag.econ.168356 ISBN : 978-99924-28-40-5

ANDI, (2018). Agroindustria. Hacia la transformación de la cadena de valor agroindustrial. ANDI . Obtenido en: http://proyectos.andi.com.co/Libro2/Paginas/assets/docs/capitulo-07.pdf

Guirao-Goris, S. (2015). Utilidad y tipos de revisión de literatura. Revista de enfermería, 9(2), 64-75. Recurperado de http://scielo.isciii.es/scielo.php?script=sci_arttext\&pid=S1988-348X2015000200002.-

Manzanelli, P., \& Schorr, M. (2012). Extranjerización y poder económico industrial en Argentina. Problemas del Desarrollo, 43(170), 39-67. doi: 10.22201/iiec.20078951e.2012.170.32135

Dourojeanni, A. (2000). Procedimientos de gestión para el desarrollo sustentable. Santiago de Chile: Cepal, Eclac.

Correa, F. (2006). Antecedentes y evolución de la economía ecológica. Semestre económico. Vol. 9 (7) 13-41).

Guirao-Goris, S. (2015). Utilidad y tipos de revisión de literatura. Revista de enfermería, 9(2), 64-75. Recurperado de http://scielo.isciii.es/scielo.php?script=sci_arttext\&pid=S1988-348X2015000200002.-

\section{Notas}

[1] Ejemplo de ecuación de búsqueda : Para WOS Tema: (("AGROINDUSTRY")) Índices=SCI-EXPANDED, SSCI, A\&HCI, ESCI Período de tiempo=Todos los años. Asimismo , Tema: (("AGROINDUSTRY"))Refinado por: Años de publicación: ( 2019 OR 2016 OR 2017 OR 2010 OR 2013 OR 2015 OR 2012 OR 2009 OR 2011 OR 2014 OR 2008 OR 2002 OR 2000 )Índices=SCI-EXPANDED, SSCI, A\&HCI, ESCI Período de tiempo=Todos los años.

[2] Véase en (Gómez,2021ª; Maldonado ,2018, Ríos, Ortiz \& Álvarez, 2005). 